\title{
FORM-FINDING ANALYSIS OF IRREGULAR TENSEGRITY STRUCTURES BY MATRIX ITERATION
}

\author{
JinYu Lu ${ }^{1,2, *}, \mathrm{Na} \mathrm{Li}^{3}$ and GanPing Shu ${ }^{1,2}$ \\ ${ }^{1}$ Key Lab of Concrete and Prestressed Concrete Structures of Ministry of Education, Nanjing 210096, China \\ ${ }^{2}$ School of Civil Engineering, Southeast University, Nanjing 210096, China \\ ${ }^{3}$ College of Electronic and Mechanical Engineering, Nanjing Forestry University, Nanjing 210037, China
}

\begin{abstract}
This paper proposed a novel form-finding method for irregular tensegrity structures base on matrix iteration. On the basis of two different forms of structural equilibrium equations, the estimated elemental self-stresses and nodal coordinates were constructed via the singular value decomposition of equilibrium matrix and eigenvalue decomposition of force density matrix, respectively. The configuration of tensegrity that satisfies the specified coordinates was determined through the iterative computation of self-stresses and nodal coordinates, and the constraint condition was introduced in the construction of the estimated nodal coordinates simultaneously. The detailed algorithm procedure was listed and the convergent criterion was also defined. In the end, several illustrated examples were given to prove the validity of the algorithm. Numerical examples and physical models showed that the proposed form-finding method was correct and efficient. The form-finding algorithm could be applied to find tensegrity structures that satisfied the given geometrical forms, and the creation of novel irregular tensegrity, as long as the topological relation and several known coordinate of nodes were given.
\end{abstract}

Keywords: Irregular tensegrity, Form-finding, Equilibrium matrix, Force density matrix, Numerical method

DOI: $10.18057 / \mathrm{IJASC} .2015 .11 .4 .7$

\section{INTRODUCTION}

Tensegrity, a kind of self-balancing system where the cables are in continuous tensional status and a few of struts are located among the cables, is light-weight but efficient, in that they could be stiffened by specified inner prestressing. Tensegrity structures have been already applied to several research fields [1,2], such as mechanical control, aerospace, biology, etc.. In addition, an alternative form of tensegrity, i.e. cable dome, has been widely used in the large span structures in contemporary architecture.

As a sort of form-sensitive structure, the superior mechanical property of tensegrity comes from its reasonable geometrical configuration. The topology, geometry and the prestressing both affect its stability and stiffness. Hence, form finding is the core of tensegrity researches [3]. The form-finding algorithms were divided into two categories by Tibert and Pellegrino [4], respectively static method and dynamic method, ranging from analytic method to force density method proposed by Schek and Linkwitz, and to dynamic relaxation method introduced earlier by Motro and Belkacem. These early form-finding algorithm gave more prominence to the research of reasonable topological relations. Accordingly, the form-finding results were mainly regular tensegrity structures, attaching little emphasis upon geometrical configuration. In recent decades, with the increasingly deeper researches, relevant scholars have made some improvements based on the classic algorithms and created several novel form-finding algorithms [5-9]. The irregular tensegrity and its geometrical configuration have achieved attention gradually. Meanwhile, the evolution theory began to be introduced to the form-finding optimization of tensegrity. The form-finding algorithms based on intelligence have some development, mainly including genetic algorithm [10-13], simulated annealing method [14], and Monte Carlos random search method [15]. 
On the basis of random thoughts, the intelligent algorithms could resolve the optimization problems covering the case of large solution space and the complex relations between objects and variables. More recently, novel form-finding algorithms were proposed to improve on the stability of the tensegrity structures [16]. However, the procedure needs so many search steps that it requires much more computation time. It should be noted that Estrada et al [7], Tran and Lee [8] introduced a form-finding algorithm based on the iterative computation of force density and coordinate. The algorithm merely needs to provide the connections of cables and struts, i.e. the topology, without the help of self-stress values and initial node coordinates, which has the advantage of faster convergence. Nonetheless, it is hard to control the nodal coordinates or the shape of form-finding results.

The paper offers a coordinate-based form-finding algorithm for irregular tensegrity. Firstly, it provides two different forms of equilibrium equations, namely, constructing self-stress with the singular value decomposition (SVD) of equilibrium matrix and nodal coordinates with eigenvalue decomposition of force density matrix. Moreover, the detailed form-finding algorithm flow is given, utilizing the nonlinear iterative computation of the self-stress and nodal coordinates, where the given coordinates are treated as constraint condition. Finally, two examples prove the effectiveness and stability of the form-finding algorithm. The topological connections of examples refer to the six-strut expanded octahedral tensegrity and six-strut truncated tetrahedral tensegrity. Furthermore, the forming method that six rigid struts hold up a flexible cable net is used in the construction process of physical model, and specific joints are designed for the implementation of model.

\section{EQUILIBRIUM EQUATION}

Without loss of generality, Figure 1 shows that node $i$ connects component $g$ and $h$ together. The internal forces are denoted as $t_{g}$ and $t_{h}$ respectively, while node $i$ is subjected to loads $p_{i x}, p_{i y}$ and $p_{i z}$. Based on the force balance relation, the equilibrium equation of node $i$ could be deduced as follows:
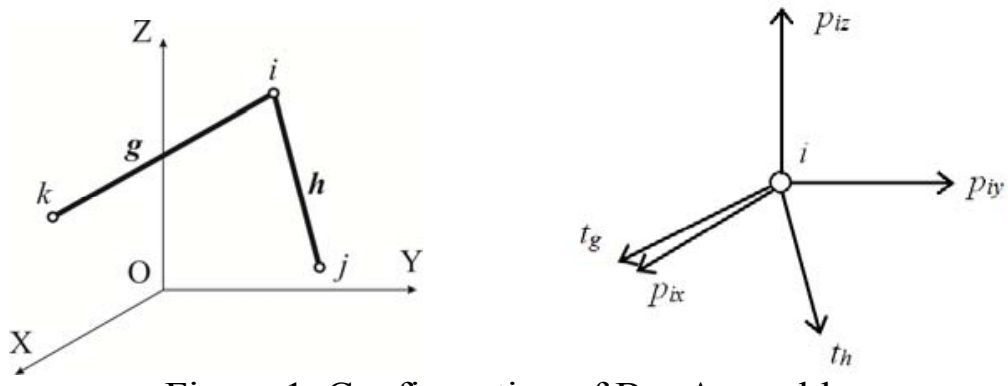

Figure 1. Configuration of Bar Assembles

$\left(x_{j}-x_{i}\right) \zeta_{h}+\left(x_{k}-x_{i}\right) \zeta_{g}=p_{i x}$

$\left(y_{j}-y_{i}\right) \zeta_{h}+\left(y_{k}-y_{i}\right) \zeta_{g}=p_{i y}$

$\left(z_{j}-z_{i}\right) \zeta_{h}+\left(z_{k}-z_{i}\right) \zeta_{g}=p_{i z}$

where $\zeta$ is force density and equals to $t / l$. Supposing that tensegrity contains $n$ nodes and $b$ elements, the global equilibrium equation can be assembled as matrix form [17]:

$\mathbf{A} \zeta=\mathbf{P}$ 
here, $\zeta=\left(\zeta_{1}, \zeta_{2}, \ldots, \zeta_{b}\right)^{\mathrm{T}}$ is the vector of force density, $\mathbf{P}=\left(\mathbf{P}_{x}, \mathbf{P}_{y}, \mathbf{P}_{z}\right)^{\mathrm{T}}$ represents the vector of nodal load and $\mathbf{P}_{x}=\left(p_{1 x}, p_{2 x}, \ldots, p_{n x}\right)^{\mathrm{T}}$ denotes the vector in $X$ degrees of freedom (DOF). The equilibrium matrix, i.e. $\mathbf{A}=\left(\mathbf{A}_{x}, \mathbf{A}_{y}, \mathbf{A}_{z}\right)^{\mathrm{T}}$, establishes the relation between nodal load and elemental internal force. Taking $X$ DOF as example, equilibrium matrix could be denoted as:

$$
\mathbf{A}_{x}=\boldsymbol{\Phi}^{\mathrm{T}} \operatorname{diag}(\boldsymbol{\Phi} \mathbf{x})
$$

in which, $\mathbf{x}=\left(x_{1}, x_{2}, \ldots, x_{n}\right)^{\mathrm{T}}$ denotes the vector of nodal coordinates, and the incidence matrix $\boldsymbol{\Phi}$ with the dimension of $b \times n$ is introduced to represent the topological connections of cables and struts. For example, the component $h$ shown in Figure 1 has initial node $i$ and terminal node $j$. In this case, $\boldsymbol{\Phi}_{h i}=1$ and $\boldsymbol{\Phi}_{h j}=-1$ should be satisfied for this element, and the other components follow the same analogy. Eq. 2 can be regarded as the equilibrium equation that takes elemental force density $\zeta$ as unknown variable. If we take nodal coordinates as unknown variable, the equation can be rewritten as:

$\mathbf{D X}=\mathbf{P}$

where $\mathbf{X}=(\mathbf{x}, \mathbf{y}, \mathbf{z})^{\mathrm{T}}$ indicates nodal coordinates vector, $\mathbf{D}$ is force density matrix, can be signified as:

$\mathbf{D}=\boldsymbol{\Phi}^{\mathrm{T}} \operatorname{diag}(\zeta) \boldsymbol{\Phi}$

Equality $\mathbf{P}=\mathbf{0}$ should be satisfied in the state of self-equilibrium for tensegrity. The following equations ought to be fulfilled:

$\mathbf{A} \zeta=\mathbf{0}$

$\mathbf{D X}=\mathbf{0}$

When the rank of equilibrium matrix is less than $b$, the structure might contain at least one self-stress mode, which is one of the necessary conditions to be a tensegrity structure.

\section{COORDINATE-BASED FORM-FINDING METHOD FOR IRREGULAR TENSEGRITY}

\subsection{Solution of Elemental Self-stress}

The singular value decomposition (SVD) is carried out for equilibrium matrix [18] A:

$\mathbf{A}=\mathbf{U S V}^{\mathbf{T}}$

Thus, orthogonal matrix $\mathbf{V}=\left(\mathbf{v}_{1}, \mathbf{v}_{2}, \ldots, \mathbf{v}_{b}\right)^{\mathrm{T}}$ is obtained. If there are $s$ modes of self-stress, it should meet the following relations [19]:

$\mathbf{A} \mathbf{V}_{s}=\mathbf{0}$

The self-stress $\zeta$ could be constructed by the vector of $\mathbf{V}_{s}=\left(\mathbf{v}_{b-s-1}, \mathbf{v}_{b-s}, \ldots, \mathbf{v}_{b}\right)^{\mathrm{T}}$. For single self-stress mode $(s=1)$, the form-finding process could use vector $\mathbf{v}_{b}$ to construct force density vector $\zeta$. Moreover, due to the principle that the struts withstand pressure and cables bear tension, if $\mathbf{v}_{b}$ does not satisfy tension-compression symbols, it is necessary to add orthogonal vectors to reconstruct the force density $\zeta[7]$. 


\subsection{Solution of Nodal Coordinate}

Nodal coordinates of tensegrity structures corresponds the nontrivial solution of Eq. 7. As force density matrix $\mathbf{D}$ is square, the eigenvalue decomposition is carried out:

$$
\mathbf{D}=\boldsymbol{\Psi} \Sigma \boldsymbol{\Psi}^{\mathrm{T}}
$$

where the orthogonal matrix $\boldsymbol{\Psi}=\left(\boldsymbol{\psi}_{1}, \boldsymbol{\psi}_{2}, \ldots, \boldsymbol{\psi}_{n}\right)^{\mathrm{T}}$. The nodal coordinates $\mathbf{X}$ could be constructed by vector $\boldsymbol{\psi}_{i}$. Supposing that the number of known nodes are $n_{0}$, the corresponding coordinate value is $\mathbf{X}_{0}$, and the remaining nodal value is $\mathbf{X}^{*} . n_{0}$ vectors are selected from $\boldsymbol{\Psi}$ to generate submatrix $\boldsymbol{\Psi}^{\prime}$. It can be blocked as $\boldsymbol{\Psi}^{\prime}=\left(\boldsymbol{\Psi}_{0}, \boldsymbol{\Psi}^{*}\right)^{\mathrm{T}}$. It gives

$$
\left(\begin{array}{ll}
\boldsymbol{\Psi}_{0} & \boldsymbol{\Psi}^{*}
\end{array}\right)^{\mathrm{T}} \boldsymbol{\alpha}=\left(\begin{array}{ll}
\mathbf{X}_{0} & \mathbf{X}^{*}
\end{array}\right)^{\mathrm{T}}
$$

$\boldsymbol{\alpha}$ in Eq. 11 is an undetermined combination coefficient. The required nodal coordinates could be solved:

$$
\mathbf{X}^{*}=\Psi^{*} \Psi_{0}^{-1} \mathbf{X}_{0}
$$

\subsection{Algorithm Procedure}

Eq. 6 and Eq. 7 establish the structural force balance relations, and the unknown variables are force density vector $\zeta$ and nodal coordinate vector $\mathbf{X}$. In addition, equilibrium matrix $\mathbf{A}$ and force density matrix $\mathbf{D}$ are assembled by $\mathbf{X}$ and $\zeta$. Thus, the nonlinear algorithm is applied to solve the problem iteratively. The detailed procedure of form-finding algorithm is presented as follows.

1) The topology and required coordinate $\mathbf{X}_{0}$ of tensegrity are given. Calculate incidence matrix $\boldsymbol{\Phi}$. Initialize each component of force density vector $\zeta_{0}$, where -1 for struts and +1 for cables.

2) Assemble force density matrix $\mathbf{D}$ according to Eq. 5 and conduct eigenvalue decomposition. Estimate the unknown nodal coordinate $\mathbf{X}^{*}$. Then modify the force density vector $\zeta$.

3) Assemble equilibrium matrix $\mathbf{A}$ according to Eq. 3 and conduct SVD. Thus, the new force density vector $\zeta$ is constructed.

4) Compute the redundant nodal force $\Delta \mathbf{P}=\mathbf{A} \zeta$ and the minimum nonzero singular value $\mathbf{S}_{r r}$ of equilibrium matrix $\mathbf{A}$. If the convergence target $\|\Delta \mathbf{P}\|_{2}<\xi_{p}$ and $\mathbf{S}_{r r}<\xi_{p}$ are both fulfilled, algorithm terminates. Otherwise, return to step 2 . Here $\xi_{P}$ is a small quantity, assuming $1 \mathrm{E}-10$. 


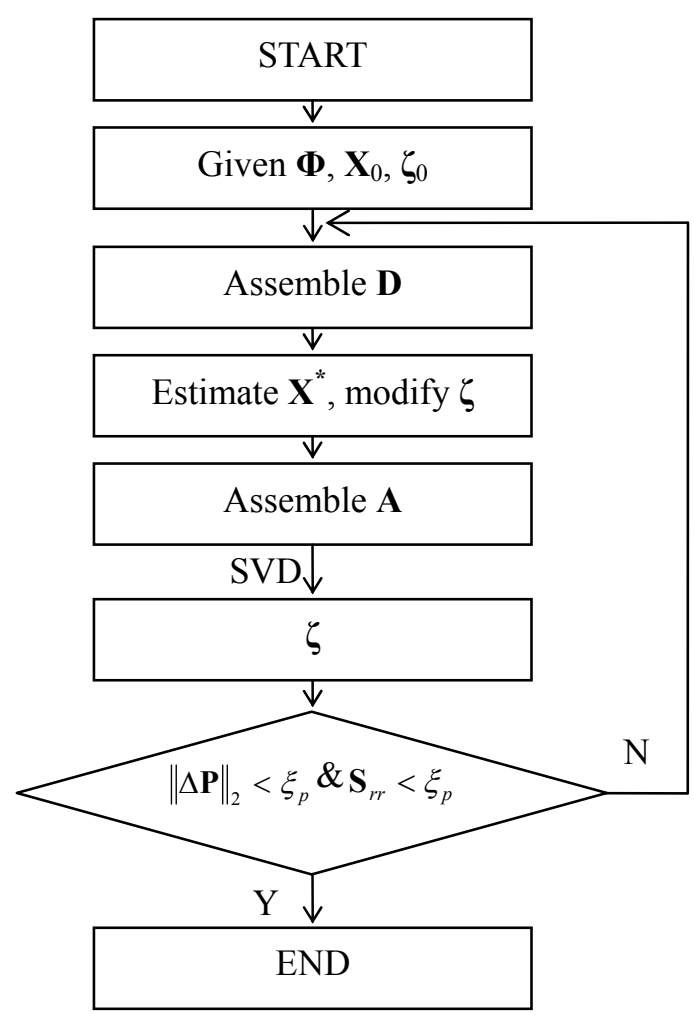

Figure 2. Algorithm Flow Chart

\section{NUMERICAL EXAMPLES}

\subsection{Form-finding of Tensegrity Structure Consists of 6 Struts and 24 Cables}

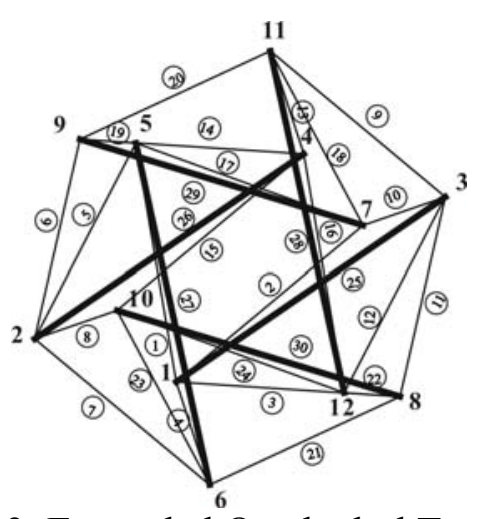

Figure 3. Expanded Octahedral Tensegrity

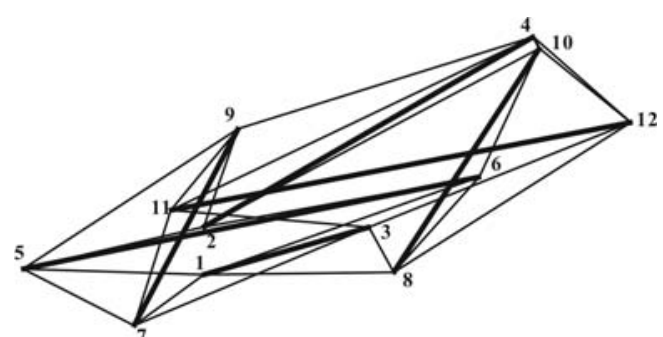

Figure 4. Computed Iirregular Tensegrity Structure

The topology of the computed structure refers to the classical expanded octahedral tensegrity shown in Figure 3. The structure has 30 elements and 12 nodes, and each node links 4 cables and 1 strut. Now the coordinates of some nodes are specified as $1(0,0,0), 2(0,100,0), 3(200,0,0)$, $4(400,300,0), 6(300,200,100)$. Supposing that $\xi_{\mathrm{p}}=1 \mathrm{E}-10$. According to the proposed algorithm, the form-finding result is presented in Figure 4. The procedure converges in 14 iterations. The computed structure contains one self-stress mode and one internal infinitesimal mechanism. It is proved to be geometrically stable since the force product is positive definite [20]. The coordinates of nodes are shown in Table 1, and Table 2 lists the internal force coefficients $t$ of cables and struts. The form-finding convergence process is illustrated in Figure 5. The physical model shown in Figure 6 are formed and stiffened by the turnbuckle installed in cable 13. Utilizing the level control of turnbuckle, the structure can achieve different stiffness. The specific joint construction is illustrated in Figure 7. 
Table 1. Nodal Coordinates of Computed Tensegrity

\begin{tabular}{ccccccccccccc}
\hline Node & 1 & 2 & 3 & 4 & 5 & 6 & 7 & 8 & 9 & 10 & 11 & 12 \\
\hline X-Value & 0.0 & 0.0 & 200.0 & 400.0 & -180. & 300.0 & -67.5 & 205.0 & 63.6 & 388.3 & -6.5 & 472.4 \\
Y-Value & 0.0 & 100.0 & 0.0 & 300.0 & -61.5 & 200.0 & -146. & 8.5 & 183.5 & 362.5 & -3.6 & 277.0 \\
Z-Value & 0.0 & 0.0 & 0.0 & 0.0 & $\begin{array}{c}-113 . \\
0\end{array}$ & 100.0 & -51.0 & 73.1 & -63.0 & 56.0 & -98.3 & 133.4 \\
\hline
\end{tabular}

Table 2. Internal Force Coefficient for Cables and Struts

\begin{tabular}{ccccccccccc}
\hline Element & 1 & 2 & 3 & 4 & 5 & 6 & 7 & 8 & 9 & 10 \\
\hline $\begin{array}{c}\text { Internal } \\
\text { force }\end{array}$ & 1.953 & 1.867 & 1.510 & 3.930 & 1.743 & 1.256 & 3.383 & 3.060 & 1.748 & 1.646 \\
Element & 11 & 12 & 13 & 14 & 15 & 16 & 17 & 18 & 19 & 20 \\
\hline $\begin{array}{c}\text { Internal } \\
\text { force }\end{array}$ & 0.677 & 1.084 & 4.006 & 2.245 & 0.880 & 0.657 & 1.322 & 1.611 & 3.640 & 2.019 \\
Element & 21 & 22 & 23 & 24 & 25 & 26 & 27 & 28 & 29 & 30 \\
\hline $\begin{array}{c}\text { Internal } \\
\text { force }\end{array}$ & 2.171 & 4.296 & 1.912 & 1.356 & -2.23 & -5.64 & -7.67 & -6.05 & -4.89 & -5.40 \\
& & & & & 7 & 8 & 2 & 7 & 5 & 8 \\
\hline
\end{tabular}

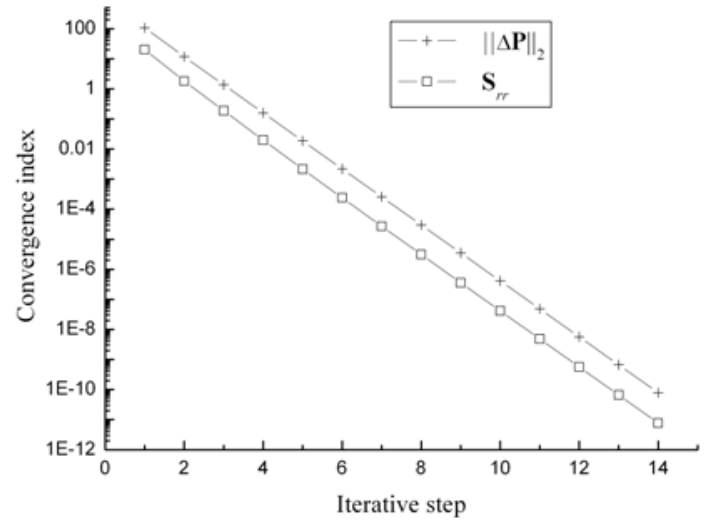

Figure 5. Iterative Procedure of Form-finding

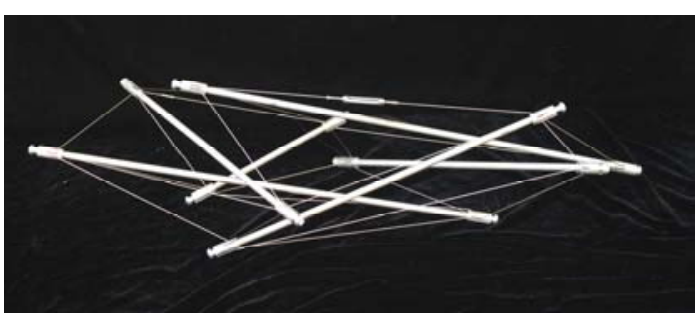

Figure 6. Physical Model of Computed Tensegrity

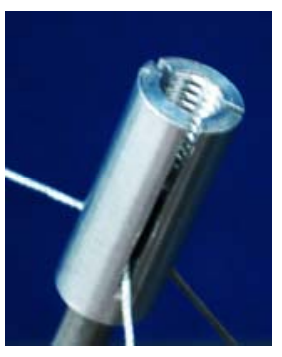

Figure 7. Joint Construction
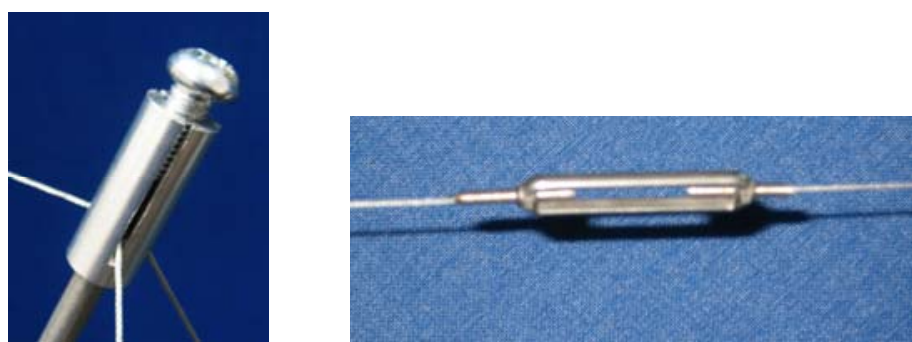

Figure 8. Turnbuckle

If the coordinates of some nodes are specified as $1(0,0,0), 3(5,0,-1), 6(0,3,0), 9(2,2,5), 12(6,2,1)$, the tensegrity configuration could be found after 14 iterations. Figure 9 and Figure 10 show the computed structural configuration and iterative procedure, respectively. And the corresponding nodal coordinates and the internal force coefficients of elements are listed in Table 1 and Table 2. 

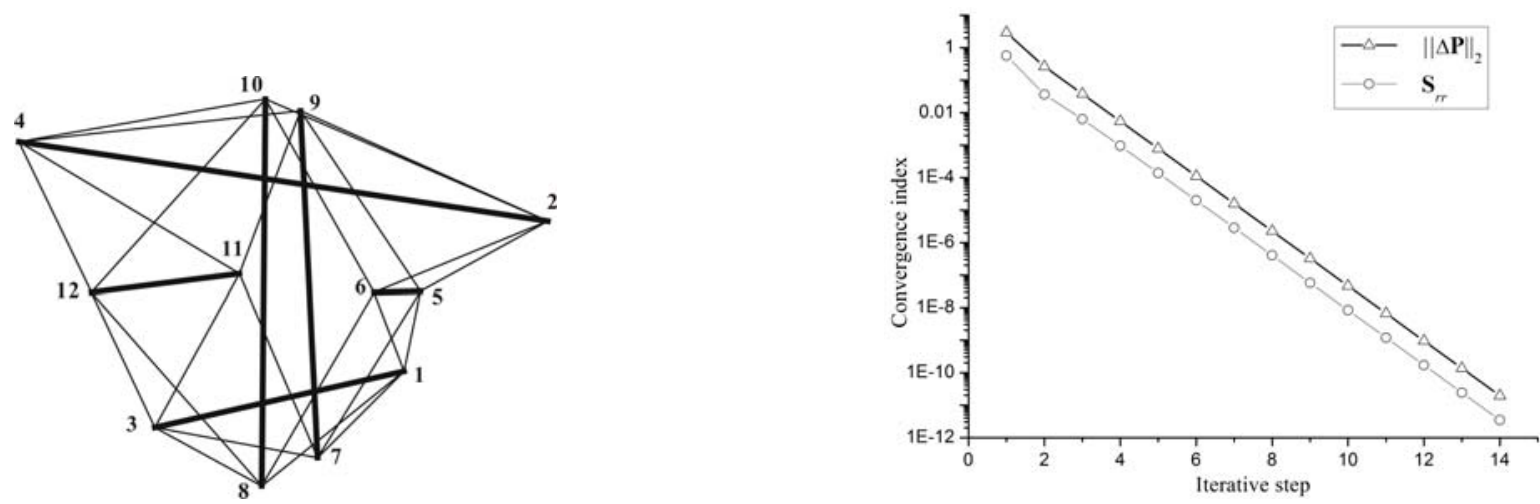

Figure 9. Computed Irregular Tensegrity Structure Figure 10. Iterative Procedure of Form-finding

Table 3. Nodal Coordinates of Computed Tensegrity

\begin{tabular}{|c|c|c|c|c|c|c|c|c|c|c|c|c|}
\hline Node & 1 & 2 & 3 & 4 & 5 & 6 & 7 & 8 & 9 & 10 & 11 & 12 \\
\hline X-Value & 0.000 & $\begin{array}{c}-3.84 \\
5\end{array}$ & 5.000 & 8.643 & $\begin{array}{c}-0.33 \\
2\end{array}$ & 0.000 & 2.199 & 2.532 & 2.000 & 2.753 & 4.071 & 6.000 \\
\hline Y-Value & 0.000 & 4.672 & 0.000 & $\begin{array}{c}-1.92 \\
0\end{array}$ & 0.464 & 3.000 & $\begin{array}{c}-2.32 \\
1\end{array}$ & 0.840 & 2.000 & 1.903 & $\begin{array}{c}-2.38 \\
9\end{array}$ & 2.000 \\
\hline Z-Value & 0.000 & 0.393 & $\begin{array}{c}-1.00 \\
0\end{array}$ & 7.106 & 1.562 & 0.000 & $\begin{array}{c}-0.46 \\
4\end{array}$ & $\begin{array}{c}-3.04 \\
8\end{array}$ & 5.000 & 5.385 & 4.007 & 1.000 \\
\hline
\end{tabular}

Table 4. Internal Force Coefficient for Cables and Struts

\begin{tabular}{ccccccccccc}
\hline Element & 1 & 2 & 3 & 4 & 5 & 6 & 7 & 8 & 9 & 10 \\
\hline $\begin{array}{c}\text { Internal } \\
\text { force }\end{array}$ & 1.868 & 3.621 & 2.634 & 1.534 & 4.184 & $\begin{array}{c}10.16 \\
5\end{array}$ & $\begin{array}{c}5.058 \\
9.726\end{array}$ & 1.896 & 1.703 \\
Element & 11 & 12 & 13 & 14 & 15 & 16 & 17 & 18 & 19 & 20 \\
\hline $\begin{array}{c}\text { Internal } \\
\text { force }\end{array}$ & 3.522 & 1.482 & 5.821 & 9.509 & 9.498 & 4.886 & 1.903 & 5.364 & 2.707 & 3.021 \\
Element & 21 & 22 & 23 & 24 & 25 & 26 & 27 & 28 & 29 & 30 \\
\hline $\begin{array}{c}\text { Internal } \\
\text { force }\end{array}$ & 4.219 & 2.558 & 4.344 & 2.239 & $\begin{array}{c}-3.81 \\
2\end{array}$ & $\begin{array}{c}-27.6 \\
83\end{array}$ & $\begin{array}{c}-2.74 \\
0\end{array}$ & $\begin{array}{c}-5.22 \\
3\end{array}$ & $\begin{array}{c}-7.80 \\
8\end{array}$ & $\begin{array}{c}-8.98 \\
0\end{array}$ \\
\hline
\end{tabular}

\subsection{Form-finding of Tensegrity Structure Consists of 6 Struts and 18 Cables}

Figure 11 shows a regular expanded octahedral tensegrity which has 6 struts and 18 cables. Each node links 1 strut and 3 cables. Nodes 1, 2, 4, 5, 7 and 8, which do not lie in the same plane, generate a space hexagon. Now it is required that the six nodes should constitute a regular hexagon of side length $200 \mathrm{~mm}$, and node 10 is located at the position of $600 \mathrm{~mm}$ above the centroid of hexagon. Figure 12 presents the calculated self-equilibrium and stable configuration whose force product is positive definite. The convergence process is shown in Figure 13. Both of the redundant nodal force and minimum nonzero singular value are lower than 1E-10 after about 190 iterative computations. The physical model is illustrated in Figure 14. 


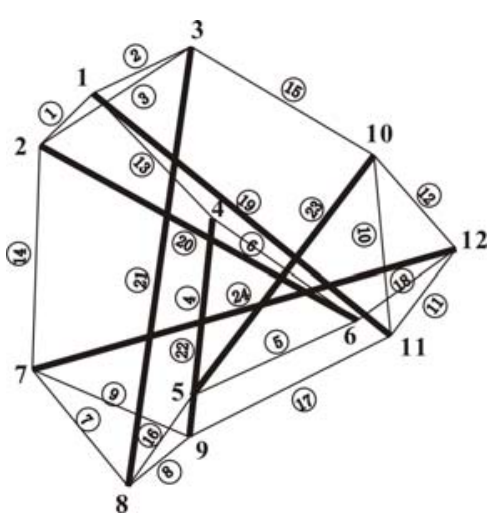

Figure 11. Expanded Octahedral Tensegrity

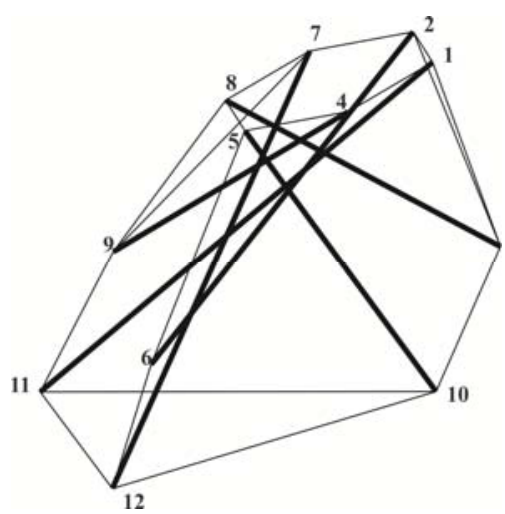

Figure 12. Computed Irregular Tensegrity Structure

Table 5. Nodal Coordinates of Computed Tensegrity

\begin{tabular}{ccccccccccccc}
\hline Node & 1 & 2 & 3 & 4 & 5 & 6 & 7 & 8 & 9 & 10 & 11 & 12 \\
\hline X-Value & 200.0 & 100.0 & 183.0 & 100.0 & -100. & -251. & -100. & -200. & -511. & 0.0 & -714. & -357. \\
& & & & & 0 & 0 & 0 & 8 & & 6 & 8 \\
Y-Value & 0.0 & 173.2 & 52.2 & -173. & -173. & -514. & 273.2 & 0.0 & 109.6 & 0.0 & -10.0 & -601. \\
& & & & 2 & 8 & 173.2 & & \\
Z-Value & 0.0 & 0.0 & 375.4 & 0.0 & 0.0 & 273.2 & 0.0 & 0.0 & 276.0 & 600.0 & 465.0 & 454.6 \\
\hline
\end{tabular}

Table 6. Internal Force Coefficient for Cables and Struts

\begin{tabular}{|c|c|c|c|c|c|c|c|c|c|c|c|c|}
\hline Element & 1 & 2 & 3 & 4 & 5 & 6 & 7 & 8 & 9 & 10 & 11 & 12 \\
\hline $\begin{array}{l}\text { Internal } \\
\text { force }\end{array}$ & 1.350 & 0.746 & 0.572 & 1.352 & 1.422 & 1.163 & 1.309 & 1.018 & 1.487 & 0.454 & 0.590 & 0.463 \\
\hline Element & 13 & 14 & 15 & 16 & 17 & 18 & 19 & 20 & 21 & 22 & 23 & 24 \\
\hline $\begin{array}{l}\text { Internal } \\
\text { force }\end{array}$ & 1.487 & 1.478 & 0.866 & 1.491 & 1.191 & 1.093 & $\begin{array}{c}-1.62 \\
9\end{array}$ & $\begin{array}{c}-1.59 \\
7\end{array}$ & $\begin{array}{c}-0.87 \\
6\end{array}$ & $\begin{array}{c}-1.56 \\
3\end{array}$ & $\begin{array}{c}-0.88 \\
4\end{array}$ & $\begin{array}{c}-1.58 \\
2\end{array}$ \\
\hline
\end{tabular}

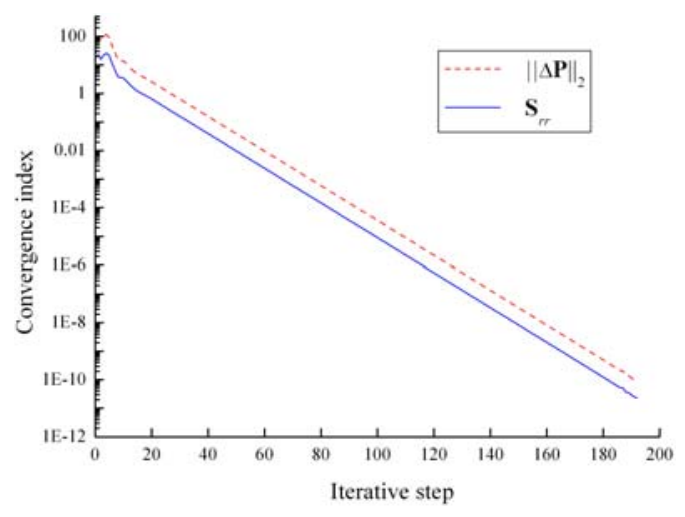

Figure 13. Iterative Procedure of Form-finding

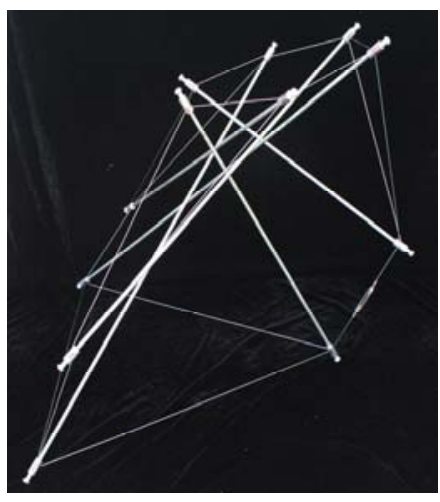

Figure 14. Physical Model of Computed Tensegrity 


\section{CONCLUSION}

This paper proposes a coordinate-based approach for the form-finding of irregular tensegrity. The algorithm resolves form-finding problems of cable-strut structures' self-equilibrium state with partly-known nodal coordinates. Based on the SVD of equilibrium matrix and eigenvalue decomposition of force density matrix, the self-stress and coordinates are constructed. And then the specified nodal coordinates are introduced as constraint conditions in the computation of coordinate values. Though a few of nonlinear iterations, the required structural configuration is determined eventually. The form-finding algorithm merely needs to provide cable-strut topological connections and some known nodal coordinates. As a result, it could be applied to find some novel irregular tensegrity structures and some tensile structures that satisfy specific geometrical shape in the foreseeable future.

\section{ACKNOWLEDGEMENT}

The research was supported by the National Natural Science Foundation of China (Grant no. 51008065, 51208263 and 51308105), the Fundamental Research Funds for the Central Universities and the Excellent Young Teachers Program of Southeast University (No. 2242014R30005), the Open Project of Jiangsu Key Laboratory of Engineering Mechanics, Southeast University, A Project Funded by the Priority Academic Program Development of Jiangsu Higher Education Institutions. The authors also thanked Ms. Xilei Zhao, Mr. Xinghua Li, Yudi Sun and Cheng Chen for their great help in making physical models.

\section{REFERENCES}

[1] Motro, R., "Tensegrity Structural Systems for the Future”, UK. Herms Science Publishing Limited, Kogan Page Limited, 2003.

[2] Skelton, R.E., Oliveira, M.C., "Tensegrity Systems", Springer Dordrecht Heidelberg London New York, 2009.

[3] Juan, S.H., Tur, J.M.M., "Tensegrity frameworks: static analysis review", Mechanism and Machine Theory, 2008, Vol. 43, No. 7, pp.859-881.

[4] Tibert, G. and Pellegrino, S., "Review of Form-finding Methods for Tensegrity Structures", International Journal of Space Structures, 2003, Vol. 18, No. 4, pp. 209-223.

[5] Zhang, L., Maurin, B. and Motro, R., "Form-finding of Nonregular Tensegrity Systems", Journal of Structural Engineering, ASCE, 2006, Vol. 132, No. 9, pp. 1435-1440.

[6] Zhang, J.Y., Ohsaki, M. and Kanno, Y., "A Direct Approach to the Design of Geometry and Forces of Tensegrity Systems”, International Journal of Solids and Structures, 2006, Vol. 43, pp. 2260-2278.

[7] Estrada, G.G., Bungartz, H.J. and Mohrdieck, C., "Numerical form Finding of Tensegrity Structure", International Journal of Solids and Structures, 2006, Vol. 43, pp. 6855-6868.

[8] Tran, H.C. and Lee, J., "Advanced form Finding of Tensegrity", Computers and Structures, 2009, Vol. 88, pp.236-247.

[9] Zhang, L.Y., Li, Y., Cao, Y.P. and Feng, X.Q., "Stiffness matrix based form-finding method of tensegrity structures", Engineering Structures, 2014, Vol. 58, pp.36-48.

[10] Paul, C., Lipson, H. and Cuevas, F.V., "Evolutionary Form-forming of Tensegrity Structures", Proceedings of the 2005 Genetic and Evolutionary Computation, Washington, USA, 2005, pp. 3-10.

[11] Rieffel, J., Cuevas, F.V. and Lipson, H., "Automated Discovery and Optimization of Large Irregular Tensegrity Structures”, Computers and Structures, 2009, Vol. 87, pp. 368-379. 
[12] $\mathrm{Xu}, \mathrm{X}$. and Luo, Y., "Form-finding of nonregular tensegrities using a genetic algorithm", Mechanics Research Communications, 2010, Vol. 37, pp. 85-91.

[13] Koohestani, K., "Form-finding of tensegrity structures via genetic algorithm", International Journal of Solids and Structures, 2012, Vol. 49, No. 5, pp. 739-747.

[14] $\mathrm{Xu}, \mathrm{X}$. and Luo, Y., "Force Finding of Tensegrity System using Simulated Annealing Algorithm", Journal of Structural Engineering, ASCE, 2010, Vol. 136, No. 8, pp. 1027-1031.

[15] Li, Y., Feng, X.Q., Cao, Y.P. and Gao, H., "A Monte Carlo Form-finding Method for Large Scale Regular and Irregular Tensegrity Structures", International Journal of Solids and Structures, 2010, Vol. 47, pp. 1888-1898.

[16] Koohestani, K., "A computational framework for the form-finding and design of tensegrity structures", Mechanics Research Communications, 2013, Vol. 54, pp.41-49.

[17] Lu, J.Y., Luo, Y.Z. and Li, N., "An Incremental Algorithm to Trace the Non-linear Equilibrium Paths of Pin-jointed Structures using the Singular Value Decomposition of the Equilibrium Matrix", Proc. IMechE Part G: J. Aerospace Engineering, 2009, Vol. 223, pp. 881-890.

[18] Lu, J.Y., Li, N. and Luo, Y.Z., "Kinematic Analysis of Planar Deployable Structures with Angulated Beams based on Equilibrium Matrix", Advances in Structural Engineering, 2011, Vol. 14, No. 6, pp. 1005-1015.

[19] Pellegrino, S. and Calliadine, C.R., "Matrix Analysis of Statically and Kinematically Indeterminate Frameworks", International Journal of Solids and Structures, 1986, Vol. 22, No. 4, pp. 409-428.

[20] Pellegrino, S., "Analysis of Pre-stressed Mechanisms", International Journal of Solids and Structures, 1990, Vol. 26, No. 12, pp. 1329-1350. 